\title{
DNA Replication and Telomere Shortening: Key Factors Related to the Production of C3-Aldehydes and the Interaction of One of them with DNA Guanine Residues
}

\author{
Dmitriev LF ${ }^{1,2^{*}}$ and Titov VN ${ }^{1}$ \\ ${ }^{1}$ Institute for Clinical Cardiology, Cardiology Research Center, Moscow, Russia \\ ${ }^{2}$ Department of Biophysics, Moscow State University, Moscow, Russia
}

"Corresponding author: Dmitriev LF, Lab of Clinical Biochemistry of Lipid Exchange, Institute for Clinical Cardiology, Moscow, Russia, E-mail: leon.d@mail.ru Rec date: Jul 05, 2014; Acc date: Sep 26, 2014; Pub date: Sep 26, 2014

Copyright: (c) 2014 Dmitriev LF, et al. This is an open-access article distributed under the terms of the creative commons attribution license, which permits unrestricted use, distribution, and reproduction in any medium, provided the original author and source are credited.

\begin{abstract}
The ketoaldehyde methylglyoxal (MG) is an aldehyde produced during aging. During cellular senescence, the ultimate and irreversible loss of replicative capacity of somatic cells takes place. The aging process needs to be explored carefully, as the well-known aging theory requires two additional ideas. One of these ideas is related to the MG-dependent loss of the hole-trapping property of the minor groove of DNA structure. The second idea pertains to local restriction and telomere shortening, which show some resemblance to the Olovnikov-Blackburn model. The ordering of such preliminary shortening is to eliminate the flawed part of the telomere and to expose the telomere to the activity of helicase.
\end{abstract}

\section{Introduction}

The synthesis of DNA in eukaryotes takes place during the S-phase of the cell cycle, and synthesis of new single-stranded DNA molecules can occur only if the parent strands are able to separate. At the origin of replication, local denaturation of DNA occurs, and the strands are mechanically separated to form two replicative forks moving in opposite directions along each strand. A number of proteins and enzymes are involved in the initiation of a replication fork.

A family of enzymes called DNA-topoisomerases furnishes the nuclease activity involved in the regulation of DNA supercoiling. In general, both prokaryotes and eukaryotic cells express several different topoisomerases, which differ in their biological functions. Indeed, the existence of a large number of different topoisomerases provides the necessary balance between the relaxed, weakly and strongly superspiraled forms of DNA. For example, DNA-topoisomerase I hydrolyzes a phosphoester linkage in one of the chains of the double helix and covalently attaches to the $5^{\prime}$ end at the point of discontinuity.

This enzyme bridges the gap in the chain and is separated from the DNA after the formation of the replication fork. Rupture of hydrogen bonds in the double-stranded DNA molecule is carried out by DNA helicase, using the energy of ATP to mechanically untwist the DNA double helix. The result is the unwinding of part of the superspiraled DNA molecule. After completion of the replication, the 5 ' ends of daughter DNA chromosome strands are incomplete due to the removal of the terminal RNA primer leaving an unreplicated gap. This takes place because the DNA polymerase responsible for filling in the gaps created by removal of the primer cannot be extended from the 3' end to the 5 ' end.

Thus, during each cycle of replication, the 5' ends of the synthesized strands would be successively shortened, a phenomenon known as the end replication problem. However, such losses do not pose a threat to the genetic information of chromosomes because this shortening of DNA occurs within telomeres. Furthermore, it is unlikely that this shortening has a real relationship with aging, as a telomere shortening of 30 nucleotide pairs per division is too small to consider seriously.

Fifty years ago, it was demonstrated by Leonard Hayflick that human diploid fibroblasts grown in culture have a finite lifespan. Since that time, innumerable experiments have been published to discover the mechanism(s) that is/are responsible for this Hayflick limit to continuous growth [1]. Taking into account the Hayflick limit, the overall number of nucleotides lost due to telomere shortening can be found as follows: 6000 nucleotides (overall telomere length)/50 cell divisions (Hayflick limit) $=120$ nucleotides shortened per division. In the majority of cases, the loss of nucleotides during basic shortening is approximately 30 nucleotides, and a shortening of 30 nucleotides per division (basic) $<<120$ nucleotides per division (total). Therefore, one can see that there are an excessive number of nucleotide pairs (approximately 90 nucleotides), and this discrepancy is not an exception to the general rule.

Two aspects of the problem related to DNA replication are under consideration in this review. We find it important to focus on the role of helicases, which catalyze the dissociation of nucleotide complexes, and on the role of nucleases, which cleave secondary structures to allow replication fork progression [2]. In addition, we take into account that oxidative stress, lipid peroxidation and protein glycation induce the formation of highly reactive endogenous aldehydes [3], including C-3 aldehydes. These aldehydes react directly with DNA and form aldehyde-derived DNA adducts, and this type of interaction and its role in DNA replication merit discussion.

\section{Aging and Telomere Shortening}

According to data $[4,5]$, the similarities between the molecular pathways that regulate aging in yeast, worms, flies and mice, together with evidence that is consistent with programmed death in salmon and other organisms, raise the possibility that programmed aging or death can also occur in higher eukaryotes. It is of interest that the mitochondrial antioxidant SkQ1 (given to rats with food) prevents the 
development of age-dependent destructive changes in both Wistar and OXYS rats [6].

Mitochondrial dysfunction is a hallmark of aging, but its causes are debated. Redox imbalance elevates ROS levels in cells, which promotes age-related diseases. In other words, altered mitochondrial function is reported to be a key underlying mechanism of many pathological states and in the aging process. More importantly, interventions that decreased ROS in cells were found to impact replicative senescence. Exposure to low ambient oxygen concentrations [7], free radical scavengers [8], over-expression of antioxidant enzymes [9] and mild, chronic mitochondrial uncoupling have been shown to decelerate telomere shortening and to extend the replicative lifespan of cells in culture. These data are all consistent with the hypothesis that one of the major contributing factors to replicative senescence is timedependent accumulation of oxidative damage. This is a key observation, because since the proposal of the free radical theory of aging [10], reports linking reactive oxygen species generation, mitochondrial function and the aging process have accumulated.

Moreover, there is evidence against a simple link between oxidative stress and aging and lifespan [11]. For example, antioxidant supplementation for nine months reduced hepatic lipid peroxidation, but DNA oxidative damage to hepatocytes and lymphocytes was unaffected. These data further question the predictions of the freeradical theory of aging and critically indicate that antioxidants can induce widely different interspecific effects on lifespan [12]. In reality, a clear concept relating the role of antioxidants in aging escapes our understanding.

Telomeres are built of identical units and grow shorter by one unit each time a cell divides, almost like a wick gradually burning down. Once they're used up, the cell withers and dies. In other words, when telomeres are critically short, cells exit the cell cycle and undergo cell senescence. Such a telomere shortening has been predicted by A. Olovnikov, and it may be uniquely determined as a programmed telomere shortening.

The telomeres are thought to prevent damage response mechanisms from recognizing the ends of linear chromosomes as double-stranded DNA breaks, including those arising when chromosomes are damaged by stress, such as ionizing radiation. In any event, it is generally accepted that telomeres are protective DNA sequences at the ends of linear chromosomes that ensure chromosomal stability [13]. They shorten with each cell division or under conditions of oxidative stress. Telomere length has been used as a "psycho-biomarker" linking stress and disease [14]. Shortened telomeres in humans are emerging as a marker of disease risk and progression, and premature mortality [15]. Different organisms use various mechanisms for telomere length homeostasis, but the details of these mechanisms are not yet completely understood.

There have been numerous studies in the last ten years correlating telomere length and mortality, Alzheimer's disease status, and longevity. On one hand, these studies are generally correlative; on the other hand, this subject bears no direct relationship to oxidative stress, and therefore on longevity or aging. Programmed telomere shortening was thoroughly examined in all its bearings, and the size by which a segment is shortened each time the cells divide is constant. As a rule, it is problematic to follow the relationship between telomere length and course of an age-related disease. At the same time, the occurrence of a relationship between telomere length and aging is too attractive to be rejected.
The hypothetical protection of genes from oxidative damage provided by the G-rich telomeric overhangs located at the ends of chromosomes, which consist, in humans, of single strands of TTAGGG sequence repeats, has been investigated in many works. Indeed, it is interesting to see that further research is being done to look at telomere length as a marker for some diseases associated with aging [16]. For this reason alone, we believe that non-programmed telomere shortening may be discussed in addition to programmed telomere shortening, and this is quite a different problem. We postulate that both these models are representations of reality, but only one of these types of telomere shortening, namely nonprogrammed telomere shortening, is associated with oxidative stress.

However, little information was available on the specific effect of the DNA sequence on the guanine oxidation property, and we still do not know whether the unique telomere sequence has special electronic properties that make it an effective hole trap and, if so, why. Recently, it was shown that the sequence dependence is very important and that modifications in the human telomeric sequence can induce crucial changes in the electronic structure of the sequence, with a concomitant increase in the ionization energy [17]. Namely, first principle MollerPlesset perturbation theory calculations reveal that the TTAGGG human telomere sequence is particularly prone to oxidation and can act as a profound hole trap as deep as a sequence of five consecutive guanines.

These theoretical results provide quantitative data indicating a high and unique efficiency of the human telomeric sequence as a trap in long-range hole migration. Today, there is no doubt that electron hole (radical cation) migration in DNA is possible and can occur over long molecular distances [18]. More precisely, breathing fluctuations in position-specific DNA base pairs are involved in regulating helicase movement into the replication fork [19]. Owing to this specific electronic structure, a replisome, which is a complex of enzymes involved in replicating DNA, attaches to the DNA, and the first of these enzymes is a helicase, which unwinds and unzips the DNA by breaking the $\mathrm{H}$ bonds between the nitrogenous bases.

It is widely accepted that in the body, most cells are unable to maintain telomere length from one division to the next, as the DNA replication machinery is unable to fully replicate the ends of chromosomes, leading to telomere shortening every time cells divide [20]. Moreover, we must really bear in mind that (i) progressive telomere shortening generates critically short telomeres that limit the replicative capacity of cells [21] and, in mice, is able to cause loss of tissue renewal capacity and progressive organ failure [22]; (ii) correlations of telomere length with healthy lifestyles suggest the role of these factors in telomere biology maintenance and its potential impact on overall health status [23].

We believe that despite positive features, the evidence for the close relationship between apoptosis and cell growth on the one hand and telomere shortening on the other is either correlative or inconclusive. The nematode is an exception to this rule, but the life span of the nematode $C$. elegans is strongly influenced by changes in the intracellular concentration of MG [24].

It is not excluded that in the case of MG crosslinking with a guanine residue, the specific electronic structure disappears and, as an inevitable consequence, the normal operation of the replisome and DNA replication become impossible. This will disturb the replicative capacity of cell. There is no way in which the annoying situation can be interconverted to the usual one except by the breaking of a telomere 
Citation: Dmitriev LF, Titov VN (2014) DNA Replication and Telomere Shortening: Key Factors Related to the Production of C3-Aldehydes and the Interaction of One of them with DNA Guanine Residues. J Gerontol Geriat Res 3: 1000175. doi:10.4172/2167-7182.1000175

Page 3 of 5

chain to retain the rest. In other words, the only way to get out of a difficult situation and start DNA replication is to use nucleases to eliminate the waste segment of the incorrect nucleotides and to have the initial electronic structure. In somatic cells, the telomere segment containing MG may be considered as a "protection lock" against superfluous cell proliferation. As for DNA replication, such a lock should be removed before the operation of the replisome and the action of helicase can initiate.

The role of programmed telomere shortening is clear, and there is no doubt about that. Nevertheless, the question arises of whether it has a bearing on longevity and aging. Instead of the simple and apprehensible statement, we propose a new approach taking into account that DNA loses its hole-trapping property because of MG cross-linking with a guanine residue. This novel concept can be described in two steps. Most cells are unable to maintain telomere length from one division to the next, as the DNA replication machinery: (i) is forced to break a segment of the telomere chain because a certain electronic structure is required to provide for helicase movement into the replication fork; (ii) is unable to fully replicate the ends of chromosomes, leading to telomere shortening every time cells divide. Thus, the principal steps of DNA replication with one or two shortenings per division are presented in Figure 1.

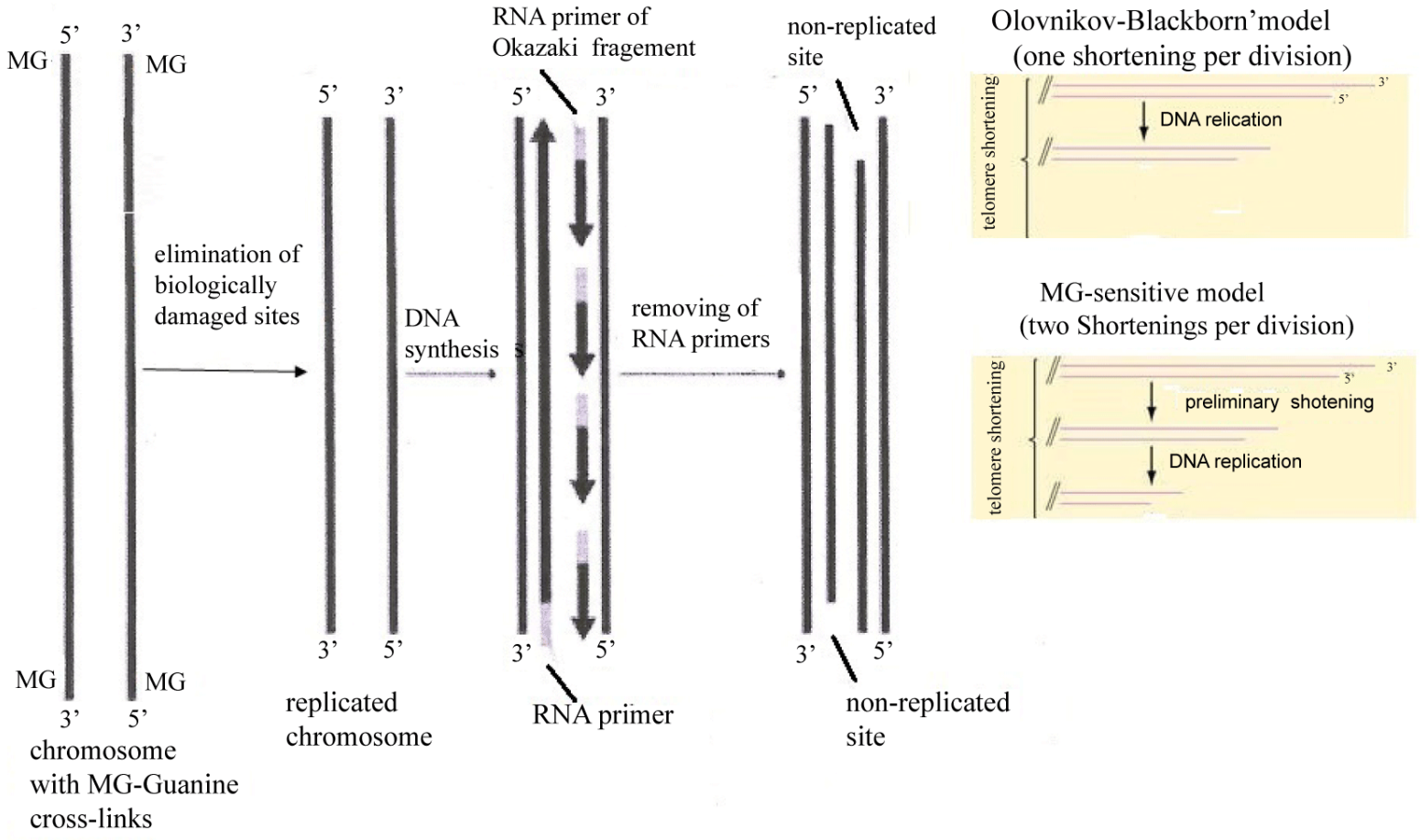

Figure 1: Shortening of telomeric DNA before and after DNA synthesis. Each of newly synthesized strands of DNA is considerably shorter than the initial one independent of basic shortening.

\section{Discussion}

In addition to the factors related to helicase activity discussed here, it should be mentioned that oxygen-free radicals, more generally known as ROS, along with lipid intermediates (LOO•) are well recognized for playing dual roles, being both deleterious and beneficial chemical species. To discuss in detail the role of the telomere in multiple aging-associated diseases, the effect of lipid intermediates and C3-aldehydes on cellular metabolism must be carefully considered.

C-3 aldehydes are formed in cells as a function of the relative contributions of O2-dependent and O2-independent (protein glycation) reactions. Because mitochondrial dysfunction involves O2dependent production of MDA and its isomer MG, there is no uncertainty about the existence of a close relationship between cellular bioenergetics and cellular senescence. The key issues are ROS production, lipid-radical cycles, ATP synthesis and ADP/ATP (Figure 2) exchange, as well as $\mathrm{C} 3$-aldehyde production, with the fatal interaction occurring between one of the aldehydes and guanine residues in the DNA. The ketoaldehyde methylglyoxal is actually an aging aldehyde, and during cellular senescence, the ultimate and irreversible loss of the replicative capacity of somatic cells takes place. 


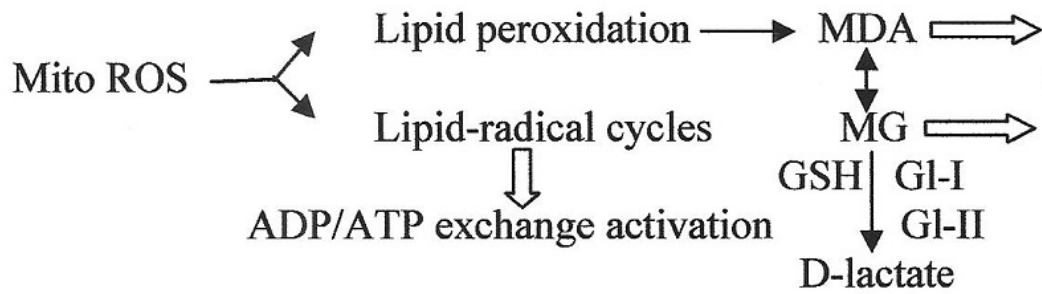

signaling to cell apoptosis machinery interaction with guanine residues telomere shortening before cell division

Figure 2: Mitochondrial dysfunction involves O2-dependent production of MDA and its isomer MG. Close relationship between cellular bioenergetics and cellular senescence includes production of C3-aldehyde with the fatal interaction between one of the aldehydes and guanine residues in the DNA.

The role of MG in cellular metabolism and cell division has been discussed for the last 45 years [25] and, in our opinion, should continue to be discussed. Our model is clearly describes cell division control in terms of MG levels negatively correlating with longevity. I believe that after being transported into the nucleus, ketoaldehyde has two distinct effects with different outcomes, and the second effect of MG is to protect DNA from telomerase attack. In reality, there is a constant production of ROS and highly reactive C-3 aldehydes, and the limited choice between moderate aging and cancer development is dictated by the convenience of the situation. It remains to be seen whether an optimum level of MG in organs and tissues is required for the appropriate control of cell division during life. In my opinion, the data from Skulachev and colleagues related to the effect of SkQ1 on mitochondrial oxidation may be considered an outstanding and encouraging development. At the same time, the level of glycated hemoglobin and/or the level of MG in plasma should be under permanent control. In reality, either an excess or a deficiency of methylglyoxal may have different types of negative consequences for cellular metabolism, and both situations are similarly undesirable.

Our data show $[26,27]$ that the normal and altered mitochondrial oxidations differ in that the first one activates lipid-radical cycles, ADP/ATP exchange and cellular metabolism [28], and second one leads to lipid peroxidation and accelerated production of MDA and MG, one of which then interacts with DNA guanine residues and is able to affect telomere length homeostasis. The latter effect is based on some experimental data $[29,30]$ and should be equally conceptualized by theoretical analysis, such as an analysis based on the principle of Moller-Plesset perturbation [17]. This means that any opinion about cellular metabolism and telomere shortening needs to be debated, and we do not know whether the afore mentioned problem is solved completely or only partially.

If we accept the non-standard idea about telomere shortening proposed here, there is nothing unusual in it, even though a considered telomere shortening takes place before DNA replication. In fact, telomere attrition represents one of the hallmarks of aging [31]. Finally, we shall have two mechanisms of telomere shortening, preliminary and basic, and one of the shortening mechanisms is a key event related to cellular metabolism. We are obliged to state that the widely accepted model (in which one telomere shortening occurs per division) remains accurate for cancer cells (in which the MG content is very low); however, the accepted model is perhaps incorrectly extended to normal somatic cells. MG presents in cancer cells in minimal quantities if present at all. For that reason, Gl-I may play a critical role in the viability of prostatic cancer cells: its activity is more than eightfold higher in prostate cancer specimens than in noncancerous specimens [32]. According to Watson [33], even though we will soon have comprehensive views on how most cancers arise and function at the genetic and biochemical levels, their 'curing' seems now to many seasoned scientists a daunting objective. We agree with Watson, because it is difficult to sustain an optimal MG content in cells for a long period of time, and the problem of inevitable aging or cancer development is tantamount to a trade-off problem.

\section{Conclusions}

1. The aging process needs to be explored carefully, and to complete the well-known aging theory, we require two additional ideas: an idea related to the MG-dependent loss of the hole-trapping property in the minor part of DNA structure tantamount to local restriction, and an idea about telomere shortening that shows some resemblance to the Olovnikov-Blackburn model. The order of such a shortening is to eliminate the flawed part of the telomere and to open it up to the activity of helicase.

2. Programmed death may not be associated only with known basic telomere shortening. This process is most likely a more complex event. In reality, telomere shortening may be reconsidered as being complemented by one more shortening - a preliminary one. We must recognize that ROS initiates C-3 aldehyde production, and one of the aldehydes interacts irreversibly with DNA guanine residues. It is most likely that cellular lifespan depends markedly on the preliminary telomere shortening, which depends on C-3 aldehyde levels.

\section{References}

1. Holliday R (2014) The commitment of human cells to senescence. Interdiscip Top Gerontol 39: 1-7.

2. León-Ortiz AM, Svendsen J, Boulton SJ (2014) Metabolism of DNA secondary structures at the eukaryotic replication fork. DNA Repair (Amst) 19: 152-162. 
Citation: Dmitriev LF, Titov VN (2014) DNA Replication and Telomere Shortening: Key Factors Related to the Production of C3-Aldehydes and the Interaction of One of them with DNA Guanine Residues. J Gerontol Geriat Res 3: 1000175. doi:10.4172/2167-7182.1000175

Page 5 of 5

3. Voulgaridou GP, Anestopoulos I, Franco R, Panayiotidis MI, Pappa A (2011) DNA damage induced by endogenous aldehydes: current state of knowledge. Mutat Res 711: 13-27.

4. Longo VD, Mitteldorf J, Skulachev VP (2005) Programmed and altruistic ageing. Nat Rev Genet 6: 866-872.

5. Balaban RS, Nemoto S, Finkel T (2005) Mitochondria, oxidants, and aging. Cell 120: 483-495.

6. Vays VB, Eldarov CM, Vangely IM, Kolosova NG, Bakeeva LE, et al. (2014) Antioxidant SkQ1 delays sarcopenia-associated damage of mitochondrial ultrastructure. Aging (Albany NY) 6: 140-148.

7. Richter $\mathrm{T}$, von Zglinicki $\mathrm{T}$ (2007) A continuous correlation between oxidative stress and telomere shortening in fibroblasts. Exp Gerontol 42: 1039-1042.

8. Saretzki G, Murphy MP, von Zglinicki T (2003) MitoQ counteracts telomere shortening and elongates lifespan of fibroblasts under mild oxidative stress. Aging Cell 2: 141-143.

9. Serra V, von Zglinicki T, Lorenz M, Saretzki G (2003) Extracellular superoxide dismutase is a major antioxidant in human fibroblasts and slows telomere shortening. J Biol Chem 278: 6824-6830.

10. Harman D (1956) Aging: a theory based on free radical and radiation chemistry. J Gerontol 11: 298-300.

11. Speakman JR, Selman C (2011) The free-radical damage theory: Accumulating evidence against a simple link of oxidative stress to ageing and lifespan. Bioessays 33: 255-259.

12. Selman C, McLaren JS, Collins AR, Duthie GG, Speakman JR (2013) Deleterious consequences of antioxidant supplementation on lifespan in a wild-derived mammal. Biol Lett 9: 20130432.

13. Blackburn EH (1991) Structure and function of telomeres. Nature 350: 569-573.

14. Epel ES (2009) Telomeres in a life-span perspective: A new "psychobiomarker"? Curr Dir Psychol Sci 18: 6-10.

15. Cawthon RM, Smith KR, O'Brien E, Sivatchenko A, Kerber RA (2003) Association between telomere length in blood and mortality in people aged 60 years or older. Lancet 361: 393-395.

16. Nakamura K, Takubo K, Izumiyama-Shimomura N, Sawabe M, Arai T, et al. (2007) Telomeric DNA length in cerebral gray and white matter is associated with longevity in individuals aged 70 years or older. Exp Gerontol 42: 944-950.

17. Cauët E (2011) Unique hole-trapping property of the human telomere sequence. J Biomol Struct Dyn 29: 557-561.

18. Makarska M, Pratviel G (2008) Long-range charge transport through double-stranded DNA mediated by manganese or iron porphyrins. J Biol Inorg Chem 13: 973-979.

19. Jose D, Weitzel SE, von Hippel PH (2012) Breathing fluctuations in position-specific DNA base pairs are involved in regulating helicase movement into the replication fork. Proc Natl Acad Sci U S A 109: 14428-14433.

20. Huffman KE, Levene SD, Tesmer VM, Shay JW, Wright WE (2000) Telomere shortening is proportional to the size of the G-rich telomeric 3'-overhang. J Biol Chem 275: 19719-19722.

21. Harley CB, Futcher AB, Greider CW (1990) Telomeres shorten during ageing of human fibroblasts. Nature 345: 458-460.

22. Armanios M, Alder JK, Parry EM, Karim B, Strong MA, et al. (2009) Short telomeres are sufficient to cause the degenerative defects associated with aging. Am J Hum Genet 85: 823-832.

23. Mirabello L, Huang WY, Wong JY, Chatterjee N, Reding D, et al. (2009) The association between leukocyte telomere length and cigarette smoking, dietary and physical variables, and risk of prostate cancer. Aging Cell 8: 405-413.

24. Hipkiss AR (2010) Proteotoxicity and the contrasting effects of oxaloacetate and glycerol on Caenorhabditis elegans life span: a role for methylglyoxal? Rejuvenation Res 13: 547-551.

25. Szent-Gyorgyi A (1968) Bioelecronics, Academic press, NY, London.

26. Dmitriev LF, Ivanova MV, Doroschuk AD (2013) Prooxidant cumene peroxide, antioxidant propyl gallate and adenine nucleotide ADP are able to affect the ATP synthesis, lipid peroxidation and oxidation of extramitochondrial $\mathrm{NADH}$ in rat liver mitochondria. Dokl Biochem Biophys 453: 292-96.

27. Agadjanyan ZS, Dugin SF, Dmitriev LF (2006) Cumene peroxide and $\mathrm{Fe}(2+)$-ascorbate-induced lipid peroxidation and effect of phosphoglucose isomerase. Mol Cell Biochem 289: 49-53.

28. Dmitriev LF (2011) Biological membranes are nanostructures that require internal heat and imaginary temperature as new, unique physiological parameters related to biological catalysts. Cell Biochem Biophys 59: 133-146.

29. Monickaraj F, Aravind S, Gokulakrishnan K, Sathishkumar C, Prabu P, et al. (2012) Accelerated aging as evidenced by increased telomere shortening and mitochondrial DNA depletion in patients with type 2 diabetes. Mol Cell Biochem 365: 343-350.

30. Murata-Kamiya N, Kamiya H (2001) Methylglyoxal, an endogenous aldehyde, crosslinks DNA polymerase and the substrate DNA. Nucleic Acids Res 29: 3433-3438.

31. López-Otín C, Blasco MA, Partridge L, Serrano M, Kroemer G (2013) The hallmarks of aging. Cell 153: 1194-1217.

32. Davidson SD, Milanesa DM, Mallouh C, Choudhury MS, Tazaki H, et al. (2002) A possible regulatory role of glyoxalase I in cell viability of human prostate cancer. Urol Res 30: 116-121.

33. Watson J (2013) Oxidants, antioxidants and the current incurability of metastatic cancers. Open Biol 3: 120144. 